\title{
Some functional measurement procedures for determining the psychophysical law
}

\author{
RICHARD S. BOGARTZ \\ University of Massachusetts, Amherst, Massachusetts 01003
}

\begin{abstract}
Various statistical procedures are reveloped for determining the psychophysical law within the context of a functional measurement approach to studying stimulus integration in perception. The specific results are limited to additive or multiplicative psychological laws, but the generalization to alternative cognitive algebras is evident. Estimation of parameters of the hypothesized psychophysical law and test of the hypothesis that the row psychophysical law is the same as the column psychophysical law in a two-factor stimulus design is considered for various possible psychophysical laws, including linear, polynomial, and power laws.
\end{abstract}

Anderson (1974) has described in detail the information integration approach to studying perception. The primary goal of this approach is establishment of psychological laws governing integration of information from sets of stimuli. Such laws are often given in the form of simple algebraic models. Given such a psychological law, stimulus scaling, response scaling, and determination of the psychophysical laws follow.

Functional measurement has been the primary methodological approach to establishment of these results. In this approach each of two or more stimulus sets constitute the factors in a factorial design, the levels of each factor being the members of the corresponding stimulus set. The separate sets of marginal means for the factorial design have been used to provide separate scales for the subjective stimulus values (Anderson, 1974). For example, if the two stimulus factors are $S$ and $T$ with levels $S_{i}$ and $T_{j}$, and if the response to the stimulus pair $\left(S_{i}, T_{j}\right)$ is $R_{i j}$ $=f_{1}\left(S_{i}\right)+f_{2}\left(T_{j}\right)=s_{i}+t_{j}$, then the row means $\overline{\mathbf{R}}_{\mathbf{i}}=\mathrm{s}_{\mathbf{i}}+\overline{\mathrm{t}}$ and the column means $\overline{\mathbf{R}}_{. j}=\overline{\mathrm{s}}+\mathrm{t}_{\mathrm{j}}$ provide interval scales for the subjective stimulus values $s_{i}$ and $t_{j}$. When $S$ and $T$ are qualitatively different variables, there will ordinarily be no interest in whether or not $f_{1}$ equals $f_{2}$. But when the integration task involves row and column physical stimulus values which may be related to the subjective values by the same psychophysical law, as for example in the bisection task or in area judgment where the heights and widths of stimulus rectangles are the row and column values, then the question of whether $f_{1}$ equals $f_{2}$ can be of great importance. The question can also be important where the same types of stimuli, perhaps even the same exact values, are present in each factor but factor denotes order of stimulus presentation, as

This work was supported by NIMH Grant MH 31139. Reprint requests should be sent to Richard S. Bogartz, Department of Psychology, University of Massachusetts, Amherst, Massachusetts 01003. for example in a time interval summing or averaging task with sequentially presented intervals (Curtis \& Rule, 1977). But even when there is no reason to be concerned with the question of equality of psychophysical laws, the problem of determining the form of each law and estimating the parameters for each function still remains.

As things presently stand, both the hypothesis testing question and the parameter estimation problem have received little attention. The prescribed approach for determining the psychophysical law (Anderson, 1974) has been plotting the marginal means in the factorial design as a function of the physical stimulus values, since the marginal means provide an interval scale of the subjective stimulus values. As a first approximation, this procedure is satisfactory, but there is considerable room for refinement. For example, if the two sets of marginal means both plot as straight lines, it might be taken as natural to pursue the question of equality of line slopes by testing whether the regression equation

$$
\overline{\mathbf{R}}=\mathrm{aU}+\mathrm{db}_{1}+(\mathbf{1}-\mathrm{d}) \mathrm{b}_{2}
$$

(in which $\bar{R}$ takes values equal to all the marginal means, $U$ is the physical stimulus value for the row or column of which $\bar{R}$ is the mean, $d$ is an indicator variable which takes the value 1 if $\bar{R}$ is a row mean and 0 if it is a column mean, $b_{1}$ and $b_{2}$ are the separate intercepts for the two lines, and $a$ is the common slope) fits as well as the equation

$$
\bar{R}=d\left(a_{1} U+b_{1}\right)+(1-d)\left(a_{2} U+b_{2}\right)
$$

(in which $a_{1}$ and $a_{2}$ are the different slopes and the other terms are as defined before). This is the standard regression approach to answering the question of whether two lines which differ in their intercepts also differ in their slopes. For I rows and J columns, the test would be based on an F distribution with 1 
and $(I+J-4)$ df. In the first result to be presented later, it will be shown that by a novel application of multiple linear regression a more powerful test of the same hypothesis can be formulated, using the responses rather than the marginal means and using an $\mathrm{F}$ distribution with 1 and ( $\mathrm{IJK}-3$ ) df ( $\mathrm{K}$ being the number of replications of the design). This new approach has a natural extension to the case where the psychophysical laws are taken to be polynomial rather than linear functions.

In general, standard multiple regression techniques are applied here, but in a new way appropriate to the functional measurement approach. This permits the use of the responses rather than the marginal means to estimate the psychophysical law parameters. The regression techniques also provide analyses of variance for testing the hypothesis that the psychophysical law for the row stimuli equals the psychophysical law for the column stimuli.

Another innovation is presented here which permits testing equality of the row and column psychophysical laws regardless of the form of the psychophysical function. It makes use of a special factorial design in which the row and column stimuli are the same. When this design is used with an additive or multiplicative psychological law, the set of row means should equal the set of column means if the two psychophysical functions are the same. The general linear model is used to develop a somewhat unusual analysis of variance for testing the hypothesis that the row means equal the column means, thereby providing an omnibus test of the hypothesis that $\mathrm{f}_{1}=\mathrm{f}_{2}$.

Various innovations in hypothesis testing follow as natural extensions of the basic results. For example, tests are developed for the hypothesis that $w_{R}$ equals $w_{C}$ in the case of the equal-weight additive psychological law $R_{i j}=w_{R} s_{i}+w_{C} t_{j}$ when various psychophysical laws are assumed, given that they are the same for row and column stimuli. Two other new tests relating to the descriptive model

$$
\mathrm{J}_{\mathrm{ij}}=\mathbf{a}\left[w \phi_{\mathrm{i}}^{\mathrm{k}}+(1-\mathrm{w}) \phi_{\mathrm{j}}^{\mathrm{k}}\right]^{\mathrm{m}}+\mathrm{b}
$$

of Curtis and Rule (1977) are also developed.

The purpose of this research, then, is to provide various statistical procedures for determining the psychophysical law or laws when the psychological law is inferred to be additive or multiplicative. The logic of these procedures then provides a basis for extension to the more general classes of models which arise in other uses of functional measurement. Two major questions are addressed here. The first is how to estimate the parameters in the psychophysical law given a hypothesis concerning the form of that law. The second question concerns testing the hypothesis that the parameters in the psychophysical law are the same for each of the two stimulus sets forming the factors of a row-by-columns stimulus design.

The results to be given are limited to a two-factor stimulus design, but the generalizations to multifactor designs will usually be obvious. Other general conditions assumed to hold throughout are that the stimulus levels in each factor are fixed effects (error variance is assumed to occur at the response side rather than the stimulus side), there are I levels of the row stimuli $S_{i}$, J levels of the column stimuli $T_{i}, K$ replications of the design, either between or within subjects, and that all effects of replications and their interactions are due to error (weaker assumptions are considered in the discussion section). A final restriction will be the assumption that, except for error, the overt behavioral response $\mathbf{R}$ is identical in value to the covert psychological response r. In general, functional measurement allows for $\mathrm{R}$ to be a monotone function of $r$, this function being referred to as the psychomotor law (Anderson, 1974, p. 283). If the psychomotor law is linear, it does not interfere with determination of the psychological law or with determining the subjective stimulus scale. If it is nonlinear, a monotone rescaling of the response may be used (e.g., Bogartz \& Wackwitz, 1971). Although some of the results to be considered here will still apply if the psychomotor law is linear or if monotone rescaling has been applied, it is simpler to consider the case where $R=r$.

\section{THE UNWEIGHTED ADDITIVE PSYCHOLOGICAL LAW: $\mathbf{R}_{\mathbf{i j}}=\mathbf{s}_{\mathbf{i}}+\mathbf{t}_{\mathbf{j}}$}

\section{The Linear Psychophysical Law}

Assuming that the usual tests for establishment of an additive psychological law have been satisfied, estimation of the slopes and intercepts in the linear psychophysical functions $s_{i}=a_{1} S_{i}+b_{1}$ and $t_{j}=$ $a_{2} T_{j}+b_{2}$, and a test of the hypothesis that $a_{1}=a_{2}$, can be accomplished using standard multiple linear regression. This is readily apparent when the psychophysical laws are inserted into the psychological law $\mathbf{R}_{\mathrm{ij}}=\mathrm{s}_{\mathrm{i}}+\mathrm{t}_{\mathrm{j}}$, and an error term is provided, giving

$$
R_{i j k}=s_{i}+t_{j}+e_{i j k}=a_{1} S_{i}+a_{2} T_{j}+b_{1}+b_{2}+e_{i j k} .
$$

(Because $b_{1}$ and $b_{2}$ cannot be estimated separately, there is no statistical test for the hypothesis that they are equal. For convenience, they are taken as equal unless otherwise stated.)

Development of the general notation for this test will be clarified by treatment of a simple example in the form of a two-factor stimulus design with three levels each of the physical stimuli $S$ and $T$, and with two replications of the design. The example $S_{i}$ values will be 1,3 , and 5 and the $T_{j}$ values will be 2,4 , and 7. Assuming that $a_{1}=a_{2}=2$ and $b_{1}=b_{2}=3$, $\mathrm{r}_{\mathrm{ij}}=2\left(\mathrm{~S}_{\mathrm{i}}+\mathrm{T}_{\mathrm{j}}\right)+6$. Assuming that the overt re- 
sponse $R_{i j k}=r_{i j}+e_{i j k}$, where $e_{i j k}$ is an independent, normally distributed error with zero mean and unknown variance, Table la shows the factorial design of $r_{i j}$ values and Table $1 b$ gives the example data consisting of two replications. The $R_{i j k}$ values in Table $1 \mathrm{~b}$ have been constructed to preserve the cell means equal to the $r_{i j}$ values in Table $1 a$ so that recovery of the parameters $a=2$ and $b=3$ can be demonstrated.

To estimate $b, a_{1}$, and $a_{2}$, the multiple regression equation

$$
\mathbf{R}=\mathbf{X}_{\mathbf{A}}^{\mathbf{T}} \boldsymbol{\beta}_{\mathbf{A}}+\mathbf{e}
$$

(under the hypothesis $H_{A}$ that $a_{1} \neq a_{2}$ ) is solved for the least squares estimates of $\boldsymbol{\beta}_{\mathrm{A}}$,

$$
\hat{\boldsymbol{\beta}}_{\mathrm{A}}=\left(\mathbf{X}_{\mathrm{A}} \mathbf{X}_{\mathrm{A}}^{\mathrm{T}}\right)^{-1} \mathbf{X}_{\mathrm{A}} \mathbf{R} \text {. }
$$

Equation 1 in expanded matrix form is just

$$
\begin{aligned}
& \begin{array}{lllll}
\mathbf{R} & \mathbf{1} & \mathbf{S} & \mathbf{T} & \boldsymbol{\beta}_{\mathrm{A}}
\end{array} \\
& {\left[\begin{array}{c}
R_{111} \\
R_{112} \\
R_{121} \\
R_{122} \\
R_{131} \\
R_{132} \\
R_{211} \\
R_{212} \\
\cdot \\
\cdot \\
\cdot \\
R_{232} \\
R_{311} \\
\cdot \\
\cdot \\
\cdot \\
R_{332}
\end{array}\right]=\left[\begin{array}{ccc}
1 & S_{1} & T_{1} \\
1 & S_{1} & T_{1} \\
1 & S_{1} & T_{2} \\
1 & S_{1} & T_{2} \\
1 & S_{1} & T_{3} \\
1 & S_{1} & T_{3} \\
1 & S_{2} & T_{1} \\
1 & S_{2} & T_{1} \\
\cdot & \cdot & \cdot \\
\cdot & \cdot & \cdot \\
\cdot & \dot{S} & T_{3} \\
1 & S_{2} & T_{1} \\
1 & S_{3} & T_{1} \\
\cdot & \cdot & \cdot \\
\cdot & \cdot & \cdot \\
\cdot & \cdot \\
a_{1} & \cdot S_{3} & T_{3}
\end{array}\right]+\left[\begin{array}{c}
e_{111} \\
e_{112} \\
e_{121} \\
e_{122} \\
e_{131} \\
e_{132} \\
e_{211} \\
e_{212} \\
\cdot \\
\cdot \\
e_{232} \\
e_{311} \\
\cdot \\
\cdot \\
\cdot \\
e_{332}
\end{array}\right]}
\end{aligned}
$$

Table 1

\begin{tabular}{|c|c|c|c|c|c|c|c|}
\hline \multicolumn{4}{|c|}{ (a) $\mathrm{r}_{\mathrm{ij}}$ Values } & \multicolumn{4}{|c|}{ (b) $\mathbf{R}_{\mathbf{i j k}}$ Values } \\
\hline \multirow[b]{2}{*}{$\mathbf{S}_{\mathbf{i}}$} & \multicolumn{3}{|c|}{$T_{j}$} & \multirow[b]{2}{*}{$\mathbf{S}_{\mathbf{i}}$} & \multicolumn{3}{|c|}{$\mathbf{T}_{\mathbf{j}}$} \\
\hline & 2 & 4 & 7 & & 2 & 4 & 7 \\
\hline 1 & 12 & 16 & 22 & 1 & $\begin{array}{l}11 \\
13\end{array}$ & $\begin{array}{l}15 \\
17\end{array}$ & $\begin{array}{l}21 \\
23\end{array}$ \\
\hline 3 & 16 & 20 & 26 & 3 & $\begin{array}{l}15 \\
17\end{array}$ & $\begin{array}{l}19 \\
21\end{array}$ & $\begin{array}{l}25 \\
27\end{array}$ \\
\hline 5 & 20 & 24 & 30 & 5 & $\begin{array}{l}19 \\
21\end{array}$ & $\begin{array}{l}23 \\
25\end{array}$ & $\begin{array}{l}29 \\
31\end{array}$ \\
\hline
\end{tabular}

Theoretical Values and Fictitious Data for the Case of Additive Psychological Law, Linear Psychophysical Law, $a_{1}=a_{2}, b_{1}=b_{2}$
Using the numerical values from Table $1 \mathrm{~b}$ gives

$$
\begin{gathered}
\mathbf{X}_{\mathrm{A}} \mathbf{X}_{\mathrm{A}}^{\mathrm{T}}=\left(\begin{array}{rrr}
18 & 54 & 78 \\
54 & 210 & 234 \\
78 & 234 & 414
\end{array}\right), \\
\left(\mathbf{X}_{\mathrm{A}} \mathbf{X}_{\mathrm{A}}^{\mathrm{T}}\right)^{-1}=\left(\begin{array}{ccc}
.490 & -.0625 & -.0570 \\
-.0625 & .0208 & 0 . \\
-.0570 & 0 . & .0132
\end{array}\right), \\
\mathbf{X}_{\mathrm{A}} \mathbf{R}=\left(\begin{array}{c}
372 \\
1212 \\
1764
\end{array}\right), \text { and } \hat{\beta}_{\mathrm{A}}=\left(\begin{array}{l}
6 \\
2 \\
2
\end{array}\right) .
\end{gathered}
$$

An analysis of variance is available to test the hypothesis $H_{A}$ against the smaller (null) hypothesis $H_{0}$ that $a_{1}=a_{2}$. Let $X_{0}^{T}=[1 S+T]$ be the $18 \times 2$ matrix

$$
\left[\begin{array}{cc}
1 & S_{1}+T_{1} \\
1 & S_{1}+T_{1} \\
1 & S_{1}+T_{2} \\
\cdot & \cdot \\
\cdot & \cdot \\
1 & S_{3}+T_{3}
\end{array}\right] .
$$

Table 2 provides the $F$ statistic for the test of $H_{A}$ vs. $H_{0}$. In Table $2, \hat{\beta}_{0}=\left(X_{0} X_{0}^{T}\right)^{-1} X_{0} R$. If the F statistic in Table 2 is nonsignificant and $H_{0}$ is accepted, then the first element in $\beta_{0}$ is $2 \hat{b}$, the least squares estimate of $2 b$, and the second is â, the least squares estimate of a. If $\mathbf{H}_{0}$ is rejected, the three elements in $\hat{\boldsymbol{\beta}}_{\mathrm{A}}$ are in order the least squares estimates of $2 b, a_{1}$, and $\mathbf{a}_{2}$.

In general, $\mathbf{R}$ will be a column vector of IJK elements, $\mathbf{X}_{\AA}^{\mathrm{T}}$ will consist of three IJK $\times 1$ column vectors: 1 , consisting of IJK $1 \mathrm{~s} ; \mathrm{S}$, consisting of JK $S_{1} s$, $J_{K} S_{2} s, \ldots, J K S_{I} s$; and $T$, consisting of I repetitions of $K T_{1} s, K T_{2} s, \ldots, K T_{J} s$; and $X_{0}^{T}$ will consist of two IJK $\times 1$ column vectors: 1 ; and $S+T$, obtained by adding $\mathbf{S}$ to $\mathbf{T}$.

\section{The Polynomial Psychophysical Law}

The estimation and hypothesis testing procedures of the previous section generalize completely to a polynomial psychophysical law

$$
\begin{aligned}
& s_{i}=a_{10}+a_{11} S_{i}+a_{12} S_{i}^{2}+a_{13} S_{i}^{3}+\ldots+a_{1 n} S_{i}^{n} \\
& t_{j}=a_{20}+a_{21} T_{j}+a_{22} T_{j}^{2}+a_{23} T_{j}^{3}+\ldots+a_{2 n} T_{j}^{n}
\end{aligned}
$$

under $\mathrm{H}_{\mathrm{A}}$ or

$$
\begin{aligned}
& s_{i}=a_{0}+a_{1} S_{i}+a_{2} S_{i}^{2}+\ldots+a_{n} S_{i}^{n} \\
& t_{j}=a_{0}+a_{1} T_{j}+a_{2} T_{j}^{2}+\ldots+a_{n} T_{j}^{n}
\end{aligned}
$$


Table 2

ANOVA Summary Table for the Test of $H_{A}: a_{1} \neq a_{2} v s . H_{0}: a_{1}=a_{2}$ for the Linear Psychophysical Law, Additive Psychological Law

\begin{tabular}{|c|c|c|c|}
\hline Source & $\mathrm{df}$ & SS & F \\
\hline $\mathrm{H}_{\mathrm{A}}$ & 3 & $\hat{\boldsymbol{\beta}}_{\mathrm{A}}^{\mathrm{T}} \mathbf{X}_{\mathrm{A}} \mathbf{R}$ & \\
\hline $\mathrm{H}_{\mathrm{o}}$ & 2 & $\hat{\boldsymbol{\beta}}_{0}^{\mathrm{T}} \mathbf{X}_{0} \mathbf{R}$ & \\
\hline $\mathrm{H}_{\mathrm{A}}$ vs. $\mathrm{H}_{0}$ & 1 & $\mathbf{S S}_{\mathbf{H}_{\mathbf{A}}}-\mathbf{S S}_{\mathrm{H}_{0}}$ & $\left(\mathrm{SS}_{\mathbf{H}_{\mathrm{A}}}-\mathrm{SS}_{\mathbf{H}_{\mathbf{0}}}\right) / \mathrm{T}$ \\
\hline Error & IJK - 3 & $\mathbf{R}^{\mathrm{T}} \mathbf{R}-\mathbf{S S}_{\mathbf{H}_{\mathbf{A}}}$ & $\left(\mathbf{R}^{\mathrm{T}} \mathbf{R}-\mathrm{SS}_{\mathbf{H}_{\mathbf{A}}}\right) /(\mathrm{IJK}-3)$ \\
\hline Total & IJK & $\mathbf{R}^{\mathrm{T}} \mathbf{R}$ & \\
\hline
\end{tabular}

Table 3

ANOVA Summary Table for the Test of $H_{0}: a_{1 i}=a_{2 i}, i=1,2, \ldots, n$ vs. $H_{A}: H_{0}$ is False for the Polynomial Psychophysical Law, Additive Psychological Law

\begin{tabular}{|c|c|c|c|c|}
\hline Source & $\underline{\mathrm{df}}$ & SS & MS & $F$ \\
\hline $\mathrm{H}_{\mathrm{A}}$ & $2 n+1$ & $\hat{\boldsymbol{\beta}}_{\mathrm{A}}^{\mathrm{T}} \mathbf{X}_{\mathrm{A}} \mathbf{R}$ & & \\
\hline $\mathbf{H}_{0}$ & $n+1$ & $\hat{\boldsymbol{\beta}}_{0}^{\top} \mathbf{X}_{0} \mathbf{R}$ & & \\
\hline $\mathrm{H}_{\mathrm{A}}$ vs. $\mathrm{H}_{\mathrm{o}}$ & n & $\mathrm{SS}_{\mathrm{A}}-\mathrm{SS}_{0}$ & $\left(\mathrm{SS}_{\mathrm{A}}-\mathrm{SS}_{0}\right) / \mathrm{n}$ & $\mathrm{MS}_{\mathrm{A} \text { vs. } 0} / \mathrm{MS}_{\mathrm{error}}$ \\
\hline Error & IJK $-2 n-1$ & $\mathbf{R}^{\mathrm{T}} \mathbf{R}-\mathrm{SS}_{\mathbf{A}}$ & $\mathrm{SS}_{\text {error }} / \mathrm{df}_{\text {error }}$ & \\
\hline Total & IJK & $\mathbf{R}^{\mathbf{T}} \mathbf{R}$ & & \\
\hline
\end{tabular}

under $\mathrm{H}_{0}$. The additive constants $\mathrm{a}_{10}$ and $\mathrm{a}_{20}$ are again assumed to be equal and estimation and hypothesis testing proceeds with respect to $\mathrm{H}_{0}$ :

$$
a_{1 i}=a_{2 i}, i=1,2, \ldots, n \text { vs. the alternative } H_{A} \text { : }
$$

$H_{0}$ is false. The vector $R$ is as described before. The matrix $\mathbf{X}_{\AA}^{T}$ is composed of the column vectors $1, \mathbf{S}$, $\mathbf{S}^{2}, \ldots, \mathbf{S}^{\mathrm{n}}, \mathbf{T}, \mathbf{T}^{2}, \ldots, \mathbf{T}^{\mathrm{n}}$, where $\mathbf{S}^{\mathrm{i}}$ is the vector of $\mathrm{ith}^{\text {th }}$ powers of the elements of the vector $\mathbf{S}$ and $\mathbf{T}^{\mathrm{i}}$ is the vector if $i$ th powers of the elements of $\mathbf{T} ; \hat{\boldsymbol{\beta}}_{\mathrm{A}}$ contains in order the least squares estimates of $2 a_{10}$, the $a_{1 i}, i=1,2, \ldots, n$, and the $a_{2 i}, i=1,2, \ldots, n$; $\mathbf{X}_{\mathbf{0}}^{\mathrm{T}}$ is composed of the column vectors $\mathbf{1}, \mathbf{S}+\mathbf{T}$, $\mathbf{S}^{2}+\mathbf{T}^{2}, \ldots, \mathbf{S}^{\mathbf{n}}+\mathbf{T}^{\mathbf{n}}$, where the vector $\mathbf{S}^{\mathbf{i}}+\mathbf{T}^{\mathbf{i}}$ is $\mathbf{S}^{\mathrm{i}}+\mathbf{T}^{\mathbf{i}}$, and $\hat{\boldsymbol{\beta}}_{0}$ contains the least squares estimates of $2 \mathrm{a}_{0}$, $a_{1}, a_{2}, \ldots, a_{n}$. Table 3 provides the $F$ statistic for the test of $\mathrm{H}_{\mathrm{A}}$ vs. $\mathrm{H}_{\mathbf{0}}$.

To exemplify the analysis in Table 3 , the data in Table 4 were constructed assuming that $\mathrm{H}_{\mathrm{A}}$ is true,

Table 4

Fictitious Data for the Case of Additive Psychological Law, Second-Order Polynomial Psychophysical Law, $a_{0}=2$, $a_{11}=3, a_{12}=2, a_{21}=4, a_{22}=3$

\begin{tabular}{cccc}
\hline & \multicolumn{3}{c}{$\mathrm{T}_{\mathbf{j}}$} \\
\cline { 2 - 4 } $\mathrm{S}_{\mathbf{i}}$ & 2 & 4 & 7 \\
\hline \multirow{2}{*}{1} & 27 & 71 & 182 \\
& 31 & 75 & 186 \\
3 & 49 & 93 & 204 \\
& 53 & 97 & 208 \\
5 & 87 & 131 & 242 \\
& 91 & 135 & 246 \\
\hline
\end{tabular}

$\mathrm{s}_{\mathrm{i}}=2+3 \mathrm{~S}_{\mathrm{i}}+2 \mathrm{~S}_{\mathrm{i}}^{2}, \mathrm{t}_{\mathrm{j}}=2+4 \mathrm{~T}_{\mathrm{j}}+3 \mathrm{~T}_{\mathrm{j}}^{2}$, and again the $S_{i}$ are $1,3,5$, and the $T_{j}$ are $2,4,7$. Under the larger hypothesis,

$$
\mathbf{R}=\left[\begin{array}{r}
27 \\
31 \\
71 \\
75 \\
182 \\
186 \\
49 \\
53 \\
93 \\
97 \\
204 \\
208 \\
87 \\
91 \\
131 \\
135 \\
242 \\
246
\end{array}\right]=\left[\begin{array}{rrrrr}
1 & 1 & 1 & 2 & 4 \\
1 & 1 & 1 & 2 & 4 \\
1 & 1 & 1 & 4 & 16 \\
1 & 1 & 1 & 4 & 16 \\
1 & 1 & 1 & 7 & 49 \\
1 & 1 & 1 & 7 & 49 \\
1 & 3 & 9 & 2 & 4 \\
1 & 3 & 9 & 2 & 4 \\
1 & 3 & 9 & 4 & 16 \\
1 & 3 & 9 & 4 & 16 \\
1 & 3 & 9 & 7 & 49 \\
1 & 3 & 9 & 7 & 49 \\
1 & 5 & 25 & 2 & 4 \\
1 & 5 & 25 & 2 & 4 \\
1 & 5 & 25 & 4 & 16 \\
1 & 5 & 25 & 4 & 16 \\
1 & 5 & 25 & 7 & 49 \\
1 & 5 & 25 & 7 & 49
\end{array}\right] \quad+\mathbf{}
$$

and $\hat{\beta}_{\mathrm{A}}=\left(\mathbf{X}_{\mathrm{A}} \mathbf{X}_{\mathrm{A}}^{\mathrm{T}}\right)^{-1} \mathbf{X}_{\mathrm{A}} \mathbf{R}=\left(\begin{array}{lllll}4 & 3 & 2 & 4 & 3\end{array}\right)^{\mathrm{T}}$, correctly recovering the parameters. Under the smaller hypothesis, $\mathrm{H}_{0}$,

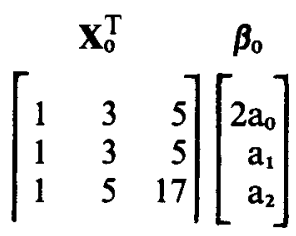




$$
\left.\mathbf{R}=\mid \begin{array}{rrr}
1 & 5 & 17 \\
1 & 8 & 50 \\
1 & 8 & 50 \\
1 & 5 & 13 \\
1 & 5 & 13 \\
1 & 7 & 25 \\
1 & 7 & 25 \\
1 & 10 & 58 \\
1 & 10 & 58 \\
1 & 7 & 29 \\
1 & 7 & 29 \\
1 & 9 & 41 \\
1 & 9 & 41 \\
1 & 12 & 74 \\
1 & 12 & 74
\end{array}\right]+\mathbf{e}
$$

and $\hat{\beta}_{0}=\left(\mathbf{X}_{0} \mathbf{X}_{0}^{\mathrm{T}}\right)^{-1} \quad \mathbf{X}_{0} \mathbf{R}=\left(\begin{array}{ll}36.82-8.02 & 4.17\end{array}\right)^{\mathrm{T}}$. Table 5 provides the results of the ANOVA described in Table 3.

Determining the order of the polynomial. Ordinarily, $\mathrm{n}$, the order of the polynomial, will not be known a priori. The appropriate procedure appears to be first settling the question of whether $a_{1 i}=a_{2 i}$, $1,2, \ldots, n$ for the largest value of $n$ to be considered and then testing whether a smaller value of $n$ is acceptable. In principle, $\mathrm{n}$ may be as large as (IJK/2) - 1 and there will still be one df for error in the $F$ test of $a_{1 i}=a_{2 i}$. In practice, $I$ would guess the initial value of $\mathrm{n}$ will rarely exceed 5 or 6 . Once the question of $a_{1 i}=a_{2 i}$ is decided, the sum of squares associated with the accepted hypothesis, $\mathbf{H}_{A}$ or $\mathbf{H}_{0}$, may be further partitioned to decide on the smallest value of $n$ which adequately describes the data. This partitioning gives rise to an $\mathrm{F}$ test following standard procedures (Mood \& Graybill, 1963, pp. 352-353, Theorem 13.6).

\section{The Power Psychophysical Law}

Somewhat different procedures are used for hypothesis testing and estimation for the power law $s=c S^{n}$. Under $H_{A}: s_{i}=c_{1} S_{i}^{n_{1}}, t_{j}=c_{2} T_{j}^{n_{2}}$; under $H_{0}: s_{i}=c S_{i}^{n}, t_{j}=c T_{j}^{n}$. To test $H_{A}$ against $H_{0}$, a factorial stimulus design is used in which the row stimuli and the column stimuli are the same. This design can of course be a subset of the total experimental design used only for hypothesis testing and

Table 5

ANOVA of the Fictitious Data in Table 4 to Test the Null

Hypothesis that a Single Second-Order Polynomial is the Psychophysical Law Against the Alternative Hypothesis that Each Stimulus Dimension Has a Different Second-Order Polynomial

\begin{tabular}{lrrrr}
\hline Source & df & \multicolumn{1}{c}{ SS } & MS & F(2,13) \\
\hline $\mathrm{H}_{\mathbf{A}}$ & 5 & 358468.00 & & \\
$\mathrm{H}_{\mathrm{o}}$ & 3 & 357650.71 & & \\
$\mathrm{H}_{\mathbf{A}}$ vs. $\mathrm{H}_{\mathrm{o}}$ & 2 & $\mathbf{8 1 7 . 2 9}$ & 408.64 & 73.76 \\
Error & 13 & 72.00 & 5.54 & \\
Total & 18 & 358540.00 & & \\
\hline
\end{tabular}

estimation. The remainder of the design then provides a means of assessing goodness of fit of the psychophysical law.

For the reduced design with row stimuli and column stimuli the same $\left(S_{i}=T_{j}\right.$ if $\left.i=j\right)$, the set of row means should equal the set of column means if $\mathbf{H}_{0}$ is true because, under $\mathrm{H}_{0}$,

$$
\begin{aligned}
& \bar{R}_{i .}=c S_{i}^{n}+c \overline{T^{n}}, \overline{T^{n}}=J^{-1} \sum_{j} T_{j}^{n}, \\
& \bar{R}_{. i}=c \overline{S^{n}}+c T_{i}^{n}, \overline{S^{n}}=I^{-1} \sum_{i} S_{i}^{n},
\end{aligned}
$$

and

$$
S_{i}=T_{i}, I=J, \overline{S^{n}}=\overline{T^{n}} .
$$

Under $\mathrm{H}_{\mathrm{A}}$, however, the set of row means and the set of column means will not be equal. Thus, the test of $\mathrm{H}_{\mathrm{A}}$ vs. $\mathrm{H}_{0}$ becomes a test of the null hypothesis that the two sets of marginal means will be equal.

To exemplify this test, consider a $3 \times 3$ factorial design with one replication per cell (for simplicity). According to the general linear model approach to the analysis of variance of an experimental design (Bogartz \& Wackwitz, 1971; Mood \& Graybill, 1963), the model without interaction is $\mathbf{R}=\mathbf{X}_{\mathrm{A}}^{\mathrm{T}} \boldsymbol{\beta}_{\mathrm{A}}+\mathbf{e}$, which, in expanded form, is

$$
\left[\begin{array}{l}
\mathbf{R}_{11} \\
\mathbf{R}_{12} \\
\mathbf{R}_{13} \\
\mathbf{R}_{21} \\
\mathbf{R}_{22} \\
\mathbf{R}_{23} \\
\mathbf{R}_{31} \\
\mathbf{R}_{32} \\
\mathbf{R}_{33}
\end{array}\right]=\left[\begin{array}{lllllll}
1 & 1 & 0 & 0 & 1 & 0 & 0 \\
1 & 1 & 0 & 0 & 0 & 1 & 0 \\
1 & 1 & 0 & 0 & 0 & 0 & 1 \\
1 & 0 & 1 & 0 & 1 & 0 & 0 \\
1 & 0 & 1 & 0 & 0 & 1 & 0 \\
1 & 0 & 1 & 0 & 0 & 0 & 1 \\
1 & 0 & 0 & 1 & 1 & 0 & 0 \\
1 & 0 & 0 & 1 & 0 & 1 & 0 \\
1 & 0 & 0 & 1 & 0 & 0 & 1
\end{array}\right]\left[\begin{array}{c}
\mu \\
\alpha_{1} \\
\alpha_{2} \\
\alpha_{3} \\
\beta_{1} \\
\beta_{2} \\
\beta_{3}
\end{array}\right]+\mathbf{e}
$$

(The side conditions $\Sigma_{i} \alpha_{i}=\Sigma_{\beta} \beta_{j}=0$ are required to augment the normal equations to full rank.)

where $\mu$ is the general mean, $\alpha_{i}$ are the row effects, and $\beta_{j}$ are the column effects. But if $H_{0}$ is true,

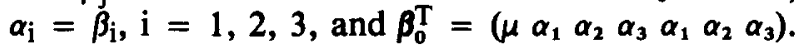
Therefore,

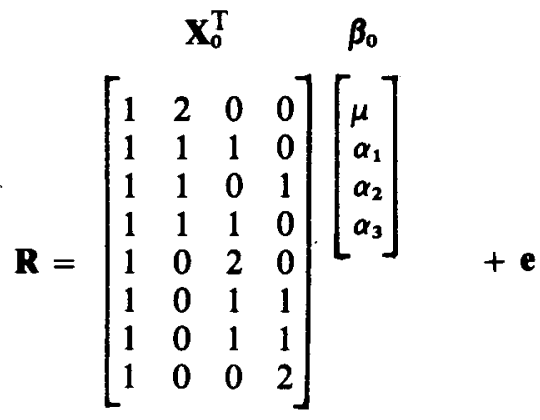


Table 6

Summary Table of the ANOVA Testing the Null Hypothesis that the Set of Row Means Equals the Set of Column Means, Assuming No Row by Column Interaction

\begin{tabular}{|c|c|c|c|c|}
\hline Source & df & SS $^{*}$ & MS & $\mathbf{F}$ \\
\hline$H_{A}$ & $21-1$ & $\left(\Sigma \mathrm{T}_{\mathrm{i}}^{2}+\sum_{\mathrm{i}} \mathrm{T}^{2},-\mathrm{T}^{2} / \mathrm{I}\right) / \mathrm{IK}$ & & \\
\hline $\mathrm{H}_{0}$ & I & {$\left[\Sigma\left(T_{i}+T_{i}\right)^{2} / 2-T^{2} / I\right] /[K$} & & \\
\hline $\mathrm{H}_{\mathrm{A}}$ vs. $\mathrm{H}_{0}$ & $1-1$ & $\mathrm{SS}_{\mathrm{A}}-\mathrm{SS}_{0}$ & $\left(\mathrm{SS}_{\mathrm{A}}-\mathrm{SS}_{0}\right) /(\mathrm{I}-1)$ & $\frac{\mathrm{MS}_{\text {A vs. } 0}}{\mathrm{MS}_{\text {error }}}$ \\
\hline Error & $I^{2} K-2 I+1$ & $\sum_{i} \sum_{j} \sum_{k} R_{i j k}^{2}-S S_{A}$ & $\mathrm{SS}_{\text {error }} / \mathrm{df}_{\text {error }}$ & \\
\hline Total & ${ }^{I^{2} \mathrm{~K}}$ & $\sum_{i} \sum_{\mathbf{j}} \sum_{\mathbf{k}} R_{i j k}^{2}$ & & \\
\hline
\end{tabular}

The reduced matrix $\mathbf{X}_{0}^{\mathrm{T}}$ is obtained by adding the column vectors in $\mathbf{X}_{\AA}^{\mathrm{T}}$ which are multiplied by the same $\alpha_{i}$ when $\mathrm{H}_{0}$ is true. This procedure of course generalizes to an $\mathrm{I} \times \mathrm{I}$ factorial design. Table 6 presents the summary table for the analysis of variance testing the hypothesis that the set of row means equals the set of column means in an $\mathrm{I} \times \mathrm{I}$ factorial design with $\mathrm{K}$ replications per cell.

If $\mathrm{H}_{\mathbf{0}}$ is rejected, the present research offers no advances concerning estimation of $c_{1}, c_{2}, n_{1}$, and $n_{2}$. The deviations of the row means from the grand mean theoretically equal $c_{1}\left(S_{i}^{n_{i}}-\overline{S^{n_{1}}}\right)$ and the corresponding column mean deviations are $c_{2}\left(T_{j}^{n_{2}}-\bar{T}^{\bar{n}_{2}}\right)$. Computer search of the parameter space seems to be the next step in estimation.

If $\mathrm{H}_{0}$ is accepted, estimation of $\mathrm{c}$ and $\mathrm{n}$ may be accomplished by setting $1 / 2\left(\overline{R_{i}}+\overline{R_{. i}}-\overline{R_{.}}\right)=$ $1 / 2\left[\left(\mathrm{cS}_{\mathrm{i}}^{\mathrm{n}}+\mathrm{c} \overline{\mathrm{T}^{\mathrm{n}}}\right)+\left(\mathrm{cT_{i } ^ { \mathrm { n } }}+\mathrm{c} \overline{\mathrm{S}^{\mathrm{n}}}\right)-\left(\mathrm{c} \overline{\mathrm{S}^{\mathrm{n}}}+\mathrm{c} \overline{\mathrm{T}^{\mathrm{n}}}\right)\right]=\mathrm{cS}_{\mathrm{i}}^{\mathrm{n}}$ since $T_{i}=S_{i}$. Taking logarithms,

$$
\ln \left[1 / 2\left(\overline{R_{i}}+\overline{R_{\cdot i}}-\overline{R_{.} .}\right)\right]=\ln c+n \ln S_{i}
$$

can be used to solve for $\ln c$ and $n$ using simple linear regression. Alternatively, to obtain estimates with more desirable statistical properties, a parameter search estimation procedure may be preferred.

If the power psychophysical law is assumed to include an additive constant so that under $\mathrm{H}_{\mathrm{A}}$, $s_{i}=c_{1} S_{i}^{n_{1}}+b_{1}$ and $t_{j}=c_{2} T_{j}^{n_{2}}+b_{2}$, the same analysis of variance test can be used to test $H_{A}$ against $\mathrm{H}_{0}: \mathrm{c}_{1}=\mathrm{c}_{2}, \mathrm{n}_{1}=\mathrm{n}_{2}$. If $\mathrm{H}_{0}$ is accepted, set

$$
\left(\overline{\mathbf{R}}_{\mathrm{i} .}+\overline{\mathbf{R}}_{. \mathrm{i}}-\overline{\mathbf{R}} . .\right) / 2=\mathrm{cS}_{\mathrm{i}}^{\mathrm{n}}+\left(\mathrm{b}_{1}+\mathrm{b}_{2}\right) / 2 .
$$

Then a parameter search can be conducted for that $\hat{\mathbf{n}}$ which maximizes the correlation of the variable on the left side of this equation with $S_{i}^{\hat{n}}$. The slope and intercept in that linear regression equation then provide the estimates $\hat{c}$ and $\left(\hat{b}_{1}+\hat{b}_{2}\right) / 2$.

\section{The Psychophysical Law of Unknown Form}

If there is no hypothesis concerning the form of the psychophysical law, one procedure would be to use the polynomial psychophysical law described before. An alternative procedure would be to use the $I \times I$ design with $S_{i}=T_{i}$ described under the power law approach. Then, if $s_{i}=f_{1}\left(S_{i}\right)$ and $t_{j}=f_{2}\left(T_{j}\right)$, the test in Table 6 for equal sets of row and column means becomes a test of $H_{0}: f_{1}=f_{2}=f$ against $H_{A}$ : $f_{1} \neq f_{2}$. If $H_{0}$ is accepted, the equation $1 / 2\left(\bar{R}_{i}+\bar{R}_{. i}-\bar{R}\right.$..) $=\mathrm{f}\left(\mathrm{S}_{\mathrm{i}}\right)$ may be used to display the form of the psychophysical function $f$ or for application of standard curvefitting procedures.

\section{THE EQUAL-WEIGHT ADDITIVE PSYCHOLOGICAL LAW $\mathbf{R}_{\mathbf{i j}}=\mathbf{w}_{\mathbf{R}} \mathbf{S}_{\mathbf{i}}+\mathbf{w}_{\mathbf{C}} \mathbf{t}_{\mathbf{j}}$}

Consideration in detail of the multitude of possible applications of the present general ideas which can arise from the various possible combinations of assumptions concerning the role of weights in the psychological law is beyond the scope of the present research. A few brief examples are provided here for the case of a weighted adding model in which the row weights all equal $\mathbf{w}_{\mathbf{R}}$ and the column weights all equal $w_{C}$ so that $R_{i j}=$ $w_{R} s_{i}+w_{C} t_{j}$.

\section{The Linear Psychophysical Law}

If $s_{i}=a_{1} S_{i}+b$ and $t_{j}=a_{2} T_{j}+b$, and if it can be assumed that $a_{1}=a_{2}$, then the test in Table 2 may be used to test the null hypothesis that $\mathrm{w}_{\mathrm{R}}=\mathrm{w}_{\mathrm{C}}$. If $\mathrm{H}_{\mathbf{0}}$ is rejected in the Table 2 test, the ratio of the last two elements in $\hat{\beta}_{A}$ will estimate the ratio of $w_{R}$ to $w_{C}$. This procedure is more general than that suggested by Anderson $(1974$, p. 224) since it does not require that the same scale values be used in each factor of the design.

\section{The Polynomial Psychophysical Law}

If

$$
S_{i}=a_{0}+\sum_{m=1}^{n} a_{1 m} S_{j}^{m} \text {, and } t_{j}=a_{0}+\sum_{m=1}^{n} a_{2 m} T_{j}^{m}
$$


and it can be assumed that $a_{1 m}=a_{2 m}, m=1,2, \ldots$, $\mathrm{n}$, then the test in Table 3 becomes a test of the null hypothesis that $w_{R}=w_{C}$. If $H_{0}$ is rejected, then the last $n$ element in $\hat{\beta}_{\mathrm{A}}$ should be linearly related to the next-to-the-last $n$ elements with an intercept of zero and a slope equal to the ratio of $w_{R}$ to $w_{C}$. Here again, it is not required that the same values be used in each factor.

\section{The Power Psychophysical Law}

In similar fashion to the procedures used in the two preceding treatments of the weights, if $s_{i}=c S_{i}^{n}$ and $t_{j}=c T_{j}^{n}$, then the test of equality of $w_{R}$ and $w_{C}$ can be performed using the ANOVA in Table 6 with a design in which the row and column stimuli are the same. If $H_{0}$ is accepted, $w_{R}=w_{C}$. If $H_{0}$ is rejected, it can be shown that the least squares estimate of the ratio $w_{C} / w_{R}$ is

$$
\hat{w}=\left(\sum_{j} R_{. j} \hat{a}_{j}\right) / \sum_{j} \hat{a}_{j}{ }^{2}
$$

where $\hat{a}_{j}=\left(\bar{R}_{j}+\hat{w} \bar{R}_{. j}-(1+\hat{w}) \bar{R} ..\right) /\left(1+\hat{w}^{2}\right)$. These two equations do not have simple explicit solutions, but $w$ is easily found by search of the w space for that $\hat{w}$ which maximizes a certain sum of squares described elsewhere (Bogartz, Note 1). A similar argument gives the same result for an unknown psychophysical function if $s_{i}=f_{1}\left(S_{i}\right), t_{j}=f_{2}\left(T_{j}\right)$, and $f_{1}=f_{2}$.

\section{THE UNWEIGHTED MULTIPLICATIVE PSYCHOLOGICAL LAW: $\mathbf{R}_{\mathbf{i j}}=s_{\mathbf{i}} \mathbf{t}_{\mathbf{j}}$}

If the multiplicative law $R_{i j}=s_{i} t_{j}$ is true, then the row-by-column interaction will be concentrated in the linear-by-linear component. If the analysis of variance indicates a significant linear-by-linear component and a nonsignificant residual component, the multiplicative law is inferentially validated and determination of the psychophysical law can proceed.

\section{The Linear Psychophysical Law}

If $s_{i}=a_{1} S_{i}+c$ and $t_{j}=a_{2} T_{j}+c$, then $R_{i j}=$ $\left(a_{1} S_{i}+c\right)\left(a_{2} T_{j}+c\right)=c^{2}+a_{1} c S_{i}+a_{2} c T_{j}+a_{1} a_{2} S_{i} T_{j}$. Again, multiple regression procedures may be applied to estimate $a_{1}$ and $a_{2}$ and to test the hypothesis $H_{0}$ : $a_{1}=a_{2}$ against $H_{A}: a_{1} \neq a_{2}$. Under $H_{A}, X_{A}^{T}=[1 S T$ ST $]$, under $H_{0}, X_{0}^{T}=[1 S+T S T]$. The vectors $S, T$, and $\mathbf{S}+\mathbf{T}$ are defined as for Table 2 , and $\mathbf{S T}$ is just the vector of products of corresponding terms, so that the $\mathrm{m}^{\text {th }}$ element in ST is the product of the $\mathrm{m}^{\text {th }}$ element in $\mathbf{S}$ times the $\mathrm{m}^{\text {th }}$ element in $\mathbf{T}$. As usual under each hypothesis $\boldsymbol{\beta}=\left(\mathbf{X} \mathbf{X T}^{\mathrm{T}}\right)^{-1} \mathbf{X} \mathbf{R}$. The appropriate ANOVA is given in Table 7 wherein a significant $F$ supports $\mathrm{H}_{A}$ over $\mathrm{H}_{0}$. If $\mathrm{H}_{0}$ is accepted, the elements in $\hat{\beta}_{0}$ estimate $c^{2}$, ac, and $a^{2}$; if $H_{A}$ is accepted, the elements in $\hat{\beta}_{A}$ estimate $c^{2}, a_{1} c, a_{2} c$, and $a_{1} a_{2}$, in which case the product of the first and fourth element should approximately equal the product of the second and third elements. Standard results on confidence intervals for the elements of $\hat{\beta}_{\mathrm{A}}$ (Mood \& Graybill, 1963, p. 351) should facilitate assessment of this expected approximate equality.

\section{The Polynomial Psychophysical Law}

Again, the obvious generalization to a polynomial of degree $n$ permits a multiple regression analysis with parameter estimation and test of the hypothesis that the row stimulus polynomial is the same as the column stimulus polynomial. Under $\mathrm{H}_{\mathrm{A}}$,

$$
s_{i}=a_{10}+\sum_{u} a_{1 u} S_{i}^{u} \text { and } t_{j}=a_{20}+\sum_{u} a_{2 u} T_{j}^{u},
$$

with $a_{10}=a_{20}$. Under $H_{0}, a_{1 u}=a_{2 u}$ for all $u=0,1$, $2, \ldots, \mathrm{n}$. In Table 8, which provides the ANOVA for this case, the matrix $\mathbf{X}_{A}^{T}$ consists of the $(n+1)^{2}$ column vectors $1, \mathbf{S}, \mathbf{S}^{2}, \ldots, \mathbf{S}^{\mathbf{n}}, \mathbf{T}, \mathbf{T}^{2}, \ldots, \mathbf{T}^{\mathbf{n}}$, $\mathbf{S T}, \mathbf{S T}^{2}, \ldots, \mathbf{S T}^{\mathrm{n}}, \mathbf{S}^{2} \mathbf{T}, \ldots, \mathbf{S}^{2} \mathbf{T}^{\mathrm{n}}, \ldots, \mathbf{S}^{\mathrm{n}} \mathbf{T}, \mathbf{S}^{\mathrm{n}} \mathbf{T}^{2}$, $\ldots, S^{n} T^{n}$, where the $m^{\text {th }}$ element of $\mathbf{S}^{u} \mathbf{T}^{\mathbf{v}}$ equals $\mathbf{S}_{m}^{u} \mathbf{T}_{m}^{v}$, with $S_{m}$ and $T_{m}$, respectively, equaling the $\mathrm{m}^{\text {th }}$ elements of $\mathbf{S}$ and $T$. The matrix $\mathbf{X}_{0}^{\mathrm{T}}$ consists of the $(n+1)(n+2) / 2$ column vectors $1, S+T, S^{2}+T^{2}$, $\ldots, \mathbf{S}^{\mathrm{n}}+\mathbf{T}^{\mathrm{n}}, \mathbf{S T}^{2}+\mathbf{S}^{2} \mathbf{T}, \mathbf{S T}^{3}+\mathbf{S}^{3} \mathbf{T}, \ldots, \mathbf{S T}^{\mathbf{n}}+\mathbf{S}^{\mathbf{n}} \mathbf{T}$, $\mathbf{S}^{2} \mathbf{T}^{2}, \mathbf{S}^{2} \mathbf{T}^{3}+\mathbf{S}^{3} \mathbf{T}^{2}, \ldots, \mathbf{S}^{2} \mathbf{T}^{\mathbf{n}}+\mathbf{S}^{\mathbf{n}} \mathbf{T}^{2}, \ldots, \mathbf{S}^{\mathbf{n}} \mathbf{T}^{\mathbf{n}}$

If $H_{0}$ is accepted, the first element of $\hat{\beta}_{0}$ is a least squares estimate of $a_{10}^{2}=a_{20}^{2}=a_{0}^{2}$, the next $n$ elements are least squares estimates of the $a_{1 u}=a_{2 u} a_{u}, u=$ $1,2, \ldots, n$, and the remaining elements estimate the products $a_{1} a_{2}, \ldots, a_{1} a_{n}, \ldots, a_{n}^{2}$. If $H_{A}$ is accepted,

Table 7

Summary Table of the ANOVA for the Multiplicative Psychological Law,

\begin{tabular}{|c|c|c|c|c|}
\hline Source & df & SS & MS & $\mathbf{F}$ \\
\hline$H_{A}$ & 4 & $\boldsymbol{\rho}_{A}^{T} \mathbf{x}_{A} \mathbf{R}$ & & \\
\hline $\mathrm{H}_{0}$ & 3 & $\mathbf{A}_{0}^{\mathrm{T}} \mathbf{X}_{0} \mathbf{R}$ & & MS \\
\hline $\mathrm{H}_{\mathrm{A}}$ vs. $\mathrm{H}_{0}$ & 1 & $S S_{A}-S S_{0}$ & $\mathrm{SS}_{\mathrm{A}}-\mathrm{SS}_{0}$ & 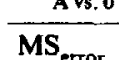 \\
\hline Error & IJK - 4 & $\mathbf{R}^{\mathbf{T}} \mathbf{R}-\mathbf{S S}_{\mathbf{A}}$ & $\mathrm{SS}_{\text {error }} / \mathrm{df}_{\text {error }}$ & \\
\hline Total & IJK & $\mathbf{R}^{\mathrm{T}} \mathbf{R}$ & & \\
\hline
\end{tabular}
Unweighted Linear Psychophysical Law 
Table 8

ANOV A for the Unweighted Multiplicative Psychological Law and Polynomial Psychophysical Law

\begin{tabular}{|c|c|c|c|c|}
\hline Source & $\mathrm{df}$ & SS & MS & $\mathbf{F}$ \\
\hline $\mathbf{H}_{\mathrm{A}}$ & $(n+1)^{2}$ & $\hat{\rho}_{\mathrm{A}}^{\mathrm{T}} \mathrm{X}_{\mathrm{A}} \mathbf{R}$ & & \\
\hline & $(n+1)(n+2) / 2$ & $\hat{\boldsymbol{\beta}}_{0}^{\mathbf{T}} \mathbf{X}_{0} \mathbf{R}$ & $2\left(\mathrm{SS}_{\mathrm{A}}-\mathrm{SS}_{0}\right)$ & $\mathrm{MS}_{\text {A vs. } 0}$ \\
\hline $\begin{array}{l}\mathrm{H}_{\mathrm{A}} \text { vs. } \mathrm{H}_{\mathrm{o}} \\
\text { Error }\end{array}$ & $\begin{array}{c}\mathrm{n}(\mathrm{n}+1) / 2 \\
1 \mathrm{JK}-(\mathrm{n}+1)^{2}\end{array}$ & $\mathbf{R}^{\mathrm{T}} \mathbf{R}-\hat{\boldsymbol{\beta}}_{\mathrm{A}}^{\mathrm{T}} \mathbf{X}_{\mathrm{A}} \mathbf{R}$ & $n(n+1)$ & $\mathbf{M S}_{\text {error }}$ \\
\hline Total & IJK & $\mathbf{R}^{\mathrm{T}} \mathbf{R}$ & & \\
\hline
\end{tabular}

the elements of $\hat{\boldsymbol{\beta}}_{\mathrm{A}}$ are, in order, the least squares estimates of $a_{0}^{2}, a_{1 u}, u=1,2, \ldots, n, a_{2 u}, u=$ $1,2, \ldots, n$ and the products $a_{11} a_{21}, a_{11} a_{22}, a_{11} a_{2 n}$, $\ldots, a_{1 n} a_{2 n}$.

\section{The Power Psychophysical Law}

It will be assumed that $s_{i}=c_{1} S_{i}^{n_{1}}, t_{j}=c_{2} T_{j}^{n_{2}}$ and that $c_{1}=c_{2}$. We seek a test of $H_{A}: n_{1} \neq n_{2}$ against $H_{0}: n_{1}=n_{2}$, and estimates of $n_{i}$. Again, the $I \times I$ design is used in which the same stimuli are used for the row and column factors $\left(S_{i}=T_{i}\right)$. Let $L_{i j}=$ in $R_{i j}$. Applying the logarithmic transformation to the $R_{i j}$ gives row and column means

$$
\overline{\mathrm{L}_{\mathrm{i} .}}=\mathrm{K}+\mathrm{n}_{1} \ln \mathrm{S}_{\mathrm{i}}+\mathrm{n}_{2} \mathrm{I}^{-1} \sum_{\mathrm{i}} 1 \mathrm{nS} \mathrm{S}_{\mathrm{i}}
$$

and

$$
\overline{\mathrm{L}}_{. \mathrm{i}}=\mathrm{K}+\mathrm{n}_{1} \mathrm{I}^{-1} \sum_{\mathrm{i}} \ln \mathrm{S}_{\mathrm{i}}+\mathrm{n}_{2} \ln \mathrm{T}_{\mathrm{i}}
$$

again assuming one score per cell $(\mathrm{K}=1)$ to simplify the notation. Since $S_{i}=T_{i}$, if $n_{1}=n_{2}$ then the row means will equal the column means. Therefore the test in Table 6 may be applied to the $L_{i j}$ to test $H_{0}$ : $n_{1}=n_{2}$ against $H_{A}: n_{1} \neq n_{2}$.

If $H_{0}$ is accepted, then $L_{i}$ and $L_{. i}$ each estimate $K+n\left(S_{i}+I^{-1} \Sigma 1 n S_{i}\right)$. The parameters $K$ and $n$ can be estimated using simple linear regression applied to the equation $1 / 2\left(\overline{\mathrm{L}}_{\mathrm{i}}+\overline{\mathrm{L}}_{\mathrm{i}}\right)=\mathrm{K}+\mathrm{n}\left(\ln \mathrm{S}_{\mathrm{i}}+\mathrm{I}^{-1} \Sigma \ln \mathrm{S}_{\mathrm{i}}\right)$. If $\mathrm{H}_{\mathrm{A}}$ is accepted, each of the $\mathrm{I}$ values $(\overline{\mathrm{L}} \mathrm{i} . \overline{\mathrm{L}} .$.$) /$ $\left(\bar{L}_{. i}-\bar{L}\right.$. .) estimates $n_{1} / n_{2}$. These can be averaged to estimate $n_{1} / n_{2}$.

If an additive constant is included in the psychophysical law so that $s_{i}=b_{1}+c_{1} S_{i}^{n_{1}}$ and $t_{j}=b_{2}+$ $c_{2} T_{j}^{n_{2}}$, then $R_{i j}=s_{i} t_{j}=b_{1} b_{2}+b_{2} c_{1} S_{i}^{n_{1}}+b_{1} c_{2} T_{j}^{n_{2}}+$ $c_{1} c_{2} S_{i}^{n_{1}} T_{j}^{n_{2}}$. It is easy to show that the row means equal the column means if $b_{1}=b_{2}=b, c_{1}=c_{2}=c$, $\mathrm{n}_{1}=\mathrm{n}_{2}=\mathrm{n}$, and $\mathrm{S}_{\mathrm{i}}=\mathrm{T}_{\mathrm{j}}$ if $\mathrm{i}=\mathrm{j}$. If the null hypothesis that the row means equal the column means is accepted, then estimation proceeds by finding that $\mathbf{n}$ which maximizes the correlation of $\left(\bar{R}_{i}+\bar{R}_{. i}-\bar{R}\right.$.. $) / 2$ with $s_{i}^{n}$, since the model asserts that $1 / 2\left(\bar{R}_{i}+\bar{R}_{i}\right.$ $-\bar{R} ..)=\left[b^{2}+b c \overline{S^{n}}-\left(c \overline{S^{n}}\right)^{2}\right]+\left(b c+c^{2} \overline{S^{n}}\right) S_{i}^{n}$ which is linear in the $S_{i}^{n}$. Given $\hat{n}$, the basic model equa- tion for $R_{i j}$ may be restated at $R_{i j}=b^{2}+b c\left(S_{i}^{\hat{n}}+S_{j}^{\hat{n}}\right)$ $+c^{2} S_{i}^{\hat{n}} S_{j}^{\hat{n}}$. The parameters $b^{2}, b c$, and $c^{2}$ may then be estimated by multiple regression methods with the estimate of bc providing a check of sorts on the estimates of $b$ and $c$ obtained as the square roots of $\hat{b}^{2}$ and $\hat{c}^{2}$.

If equality of row and column means is rejected, the separate maximization of the correlation of the $\bar{R}_{i}$ with the $S_{i}^{n_{1}}$ and the $\bar{R}_{\text {.j }}$ with the $T_{j}^{n_{2}}$ can be used to find $\hat{n}_{1}$ and $\hat{n}_{2}$. These can then be inserted into the equation for $R_{i j}$, and the products $b_{1} b_{2}, b_{2} c_{1}, b_{1} c_{2}$, and $c_{1} c_{2}$ can be estimated using multiple regression procedures.

\section{The Psychophysical Law of Unknown Form}

Here, as in the case of the additive psychological law, if there is no a priori hypothesis concerning the form of the psychophysical law, either the polynomial or the $I \times I$ design with $S_{i}=T_{i}$ can be used. For the latter case, the $L_{i j}=1 n R_{i j}$ can be used. For $R_{i j}=$ $\mathrm{f}_{1}\left(\mathrm{~S}_{\mathrm{i}}\right) \mathrm{f}_{2}\left(\mathrm{~T}_{\mathrm{j}}\right), \ln \mathrm{R}_{\mathrm{ij}}=\mathrm{L}_{\mathrm{ij}}=\ln \mathrm{f}_{1}\left(\mathrm{~S}_{\mathrm{i}}\right)+\ln \mathrm{f}_{2}\left(\mathrm{~T}_{\mathrm{j}}\right), \overrightarrow{\mathrm{L}}_{\mathrm{i}}=$ $\operatorname{lnf}\left(S_{i}\right)+\overline{\operatorname{lnf}_{2}}, \bar{L}_{. i}=\overline{\operatorname{lnf}_{1}}+\operatorname{lnf} f_{2}\left(S_{i}\right)$, and $\bar{L} . .=$ $\overline{\operatorname{lnf} f_{1}}+\overline{\operatorname{lnf}}$. If $H_{0}: f_{1}=f_{2}=f$ is accepted, then $1 / 2\left(\overline{\mathrm{L}}_{\mathrm{i}}+\overline{\mathrm{L}}_{. \mathrm{i}}-\overline{\mathrm{L}}_{. .}\right)$estimates $\ln \mathrm{f}\left(\mathrm{S}_{\mathrm{i}}\right)$ and $\exp \left[1 / 2\left(\overline{\mathrm{L}}_{\mathrm{i}}\right.\right.$. $\left.\left.+\bar{L}_{. i}-L_{. .}\right)\right]$estimates $f\left(S_{i}\right)$.

\section{SOME RESULTS FOR A MORE ELABORATE POWER LAW FORMULATION}

Curtis and Rule (1977) proposed a general descriptive model for the relation between time duration judgments and time interval stimuli of the form $\mathbf{J}_{\mathbf{i j}}$ $=a\left[w \phi_{u}^{k}+(1-w) \phi_{j}^{k}\right]^{m}+b$, where $J_{i j}$ is a judgment of the joint effect of two durations with measures $\phi_{i}$ and $\phi_{j}$, and $w$ and $(1-w)$ weight the first and second stimulus of a series, reflecting the possible presence of time-order errors. If no such errors occur, $w=$ $1-w=.5$. Among other applications, Curtis and Rule used this model for judgments of the average of two sequentially presented intervals. They found that $\mathrm{J}_{\mathrm{ij}}=.95\left[.51 \phi_{\mathrm{i}}{ }^{1.94}+.49 \phi_{\mathrm{j}}{ }^{1.94}\right]^{.49}+.94$, using the GaussNewton method (Hartley, 1961) to estimate the parameters.

Because Curtis and Rule used a factorial stimulus design in which the row stimuli equaled the column stimuli, the null hypothesis that $\mathrm{w}=1-\mathrm{w}$ can be 
tested statistically (although, for their specific results, giving estimates of $w=.51$ and $1-w=.49$, there would be little point). If $w=1-w$, their formulation predicts that the row means will equal the column means. That is, for row $\mathrm{n}$ the mean is

$$
\overline{J_{n} .}=J^{-1} \sum_{j=1}^{J} a\left(.5 \phi_{n}^{k}+.5 \phi_{j}^{k}\right)^{m}+b
$$

and for column $\mathrm{n}$ the mean is

$$
\bar{J}_{. n}=I^{-1} \sum_{i=1}^{I} a\left(.5 \phi_{i}^{k}+.5 \phi_{n}^{k}\right)^{m}+b .
$$

The quantities $\bar{J}_{\mathrm{n}}$ and $\bar{J}_{\mathrm{n}}$ are equal, since $\mathrm{I}=\mathrm{J}$ is the number of stimuli in each row and each column of the design and $\phi_{i}=\phi_{j}$ if $i=j$. However, if $w \neq 1-w$, $\overline{J_{n}} \neq \overline{J_{. n}}$, in general. Thus, the test in Table 6 can be applied to this formulation to test $\mathrm{H}_{0}: \mathrm{w}=1-\mathrm{w}=.5$.

Curtis and Rule also had their subjects rate the magnitudes of the individual intervals on a scale of 1 to 11 . Previous research had shown that the relation of such category ratings to stimulus measures is well described by a power function of the form $\mathrm{J}=\mathbf{a} \phi^{\mathrm{n}}+\mathbf{b}$. Curtis and Rule argued that their model predicted that judgments of a single stimulus duration should be described by the function $\mathrm{J}=$ $a \phi^{k m}+b$ since for a single stimulus duration, $\phi_{j}$ is zero. Thus, they expected that the estimate of $n$ for ratings of the single stimulus duration should be approximately equal to the product of the estimates of $k$ and $\mathrm{m}$. Their results generally supported this prediction quite well. For judgments of average duration, their estimate of $\mathrm{k} \times \mathrm{m}$ was 1.16 and their estimate of $\mathrm{n}$ was 1.19. When the agreement is this close, there is little need for statistical tests, but in general it would be desirable to test the null hypothesis that $\mathrm{k} \times \mathrm{m}=\mathrm{n}$. A test of this hypothesis is possible based on the $\mathrm{J}_{\mathrm{ii}}$ data. Theoretically,

$$
J_{i i}=a\left[w \phi_{i}^{k}+(1-w) \phi_{i}^{k}\right]^{m}+b=a \phi_{i}^{k m}+b .
$$

Therefore, judgments of the average of two identical durations will equal the judgments of one of the durations if $\mathrm{k} \times \mathrm{m}=\mathrm{n}$. Judgments of the averages and of the single stimuli lend themselves to an obvious analysis of variance test of the null hypothesis that the differences between the two types of scores are zero and that there are no effects due to stimuli or interactions with stimuli.

The modified Gauss-Newton parameter estimation procedure used by Curtis and Rule is fairly complicated. A somewhat simpler procedure will be developed here. It was observed above that $\mathrm{J}_{\mathrm{ii}}=\mathrm{a} \phi_{\mathrm{i}}^{\mathrm{km}}+\mathrm{b}$ so that $J_{i i}$ is a linear function of $\phi_{i}^{k m}$. A simple parameter space search for the exponent $u$ which maximizes the correlation of the $J_{i i}$ with the $\phi_{i}^{u}$ provides an estimate $\mathbf{u}$ of the product $\mathbf{k} \times \mathbf{m}$. Given this value of $\hat{\mathbf{u}}$, the slope and intercept of the resulting regression line may be taken as the estimates $\hat{a}$ and $\hat{b}$. Using these estimates, the $J_{i j}$ may be linearly transformed to $K_{i j}$ $=\left(\mathrm{J}_{\mathrm{ij}}-\hat{\mathrm{b}}\right) / \hat{\mathrm{a}}=\left[\mathrm{w} \phi_{1}^{\mathrm{k}}+(1-\mathrm{w}) \phi_{\mathrm{j}}^{\mathrm{k}}\right]^{\mathrm{m}}$. This implies additivity for the $\mathrm{K}_{\mathrm{ij}}^{-\mathrm{m}}$. By parameter search locate that $1 / \hat{m}$ to which the $K_{i j}$ must be raised in order to minimize the interaction in the $K_{i j}^{-m}$ values. Then $\hat{\mathbf{m}}^{-1} \times \hat{\mathbf{u}}=\hat{\mathbf{k}}$ provides an estimate of $\mathbf{k}$.

The remaining parameter $w$ may now easily be estimated. Let $L_{i j}=K_{i j}-\hat{m}$. Then

$$
\begin{aligned}
& \overline{\mathrm{L}}_{\mathrm{i} .}=w \phi_{\mathrm{i}}^{\hat{\mathrm{k}}}+(1-w) \phi^{\overline{\mathrm{k}}}, \\
& \overline{\mathrm{L}}_{. \mathrm{j}}=w \bar{\phi}^{\overline{\mathrm{k}}}+(1-w) \phi_{\mathrm{j}}^{\hat{\mathrm{k}}},
\end{aligned}
$$

and

$$
\overline{\mathrm{L}} . .=w \overline{\phi^{\hat{k}}}+(1-w) \overline{\phi^{\bar{k}}}=\overline{\phi^{\bar{k}}} .
$$

The row effects are

$$
\overline{\mathrm{Li}} .-\overline{\mathrm{L}} . .=w\left(\phi_{\mathrm{i}}^{\hat{\mathrm{k}}}-\bar{\phi}^{\overline{\mathrm{k}}}\right)
$$

and the column effects are

$$
\begin{aligned}
\overline{\mathrm{L}}_{\cdot j}-\overline{\mathrm{L}} \ldots & =(1-w)\left(\phi_{j}^{\hat{\mathrm{k}}}-\overline{\phi^{\hat{k}}}\right) \\
& =w\left(\phi^{\overline{\mathrm{k}}}-\phi_{\mathrm{j}}^{\hat{\mathrm{k}}}\right)+\phi_{\mathrm{j}}^{\hat{\mathrm{k}}}-\overline{\phi^{\overline{\mathrm{k}}}},
\end{aligned}
$$

giving

$$
\overline{\mathrm{L}}_{\cdot \mathrm{j}}-\overline{\mathrm{L}} \ldots+\phi^{\overline{\hat{k}}}-\phi_{\mathrm{j}}^{\hat{\mathrm{k}}}=w\left(\phi^{\overline{\mathrm{k}}}-\phi_{\mathrm{j}}^{\hat{\mathrm{k}}}\right) .
$$

Both equations are of the form $y=w x$, so the least squares solution for $w$, given the estimates for the remaining parameters, is just

$$
\begin{gathered}
\hat{\mathbf{w}}=\left[\sum_{i}\left(\overline{L_{i}}-\bar{L} \ldots\right)\left(\phi_{i}^{\hat{k}}-\bar{\phi}^{\bar{k}}\right)+\sum_{j}\left(\bar{L}_{\cdot j}-\bar{L} . .\right.\right. \\
\left.\left.+\overline{\phi^{\hat{k}}}-\phi_{j}^{k}\right)\left(\phi^{\hat{k}}-\phi_{j}^{\hat{k}}\right)\right] / 2 \sum_{j}\left(\phi_{j}^{\hat{k}}-\phi^{\bar{k}}\right)^{2} .
\end{gathered}
$$

\section{DISCUSSION}

The results presented here are easily extrapolated to more general models. For example, Anderson has defined a class of multilinear models which use adding and multiplying models as building blocks (Anderson, 1974, p. 264). Two examples of these would be $R_{i j k}=s_{i} t_{j} u_{k}$ and $R_{i j k}=s_{i}\left(t_{j}+u_{k}\right)$. Anderson defines these models generically as a sum of products of the stimulus factors such that each factor occurs 
in each product in all-or-none fashion. If it were hypothesized that in the case of the second example model the psychophysical law was linear, then $R_{i j k}=$ $\left(a_{1} S_{i}+b\right)\left(a_{2} T_{j}+b\right)+\left(a_{1} S_{j}+b\right)\left(a_{3} U_{k}+b\right)$ and the obvious extensions of the present results could be used to test the null hypothesis that $a_{1}=a_{2}=a_{3}$.

The general approach presented here appears to have implications for more detailed analysis of the models which use weights, although these implications have not been pursued here in detail. Functional measurement allows for the possibility that the weight assigned to the psychological value of a stimulus may depend upon the stimulus value.Thus, for example, the algebraic model might be $R_{i j}=w_{R_{i}} s_{i}$ $+w_{C_{j}} t_{j}$. I am not aware of any name that has been given to the law that relates the physical value of the stimulus to the numerical value of the weight assigned to the psychological value of that stimulus. Still, such a law appears to be of comparable interest to the psychophysical law relating the physical stimulus value to the psychological stimulus value. The present approach should be of some use in the study of such a law.

For example, suppose that the psychophysical law is taken to be linear, and that it is further assumed that the same psychophysical law applies to both the row stimuli and the column stimuli in a two-factor stimulus design. It may be of further interest to decide whether the row weight assigned to $s_{i}$ equals the column weight assigned to $t_{j}$ if $s_{i}=t_{j}$. Provided that this is true, it may also be of interest to determine whether there is any difference between the weights assigned to one row and the weights assigned to another. To answer the first question, the $I \times I$ design with the same row and column stimuli could be used. The test would be based on the difference between the model that assumes different weights for rows than for columns and the model which assumes the same weights. Without getting into the details of the matrices $\mathbf{X}_{\mathrm{A}}^{\mathrm{T}}$ and $\mathbf{X}_{0}^{\mathrm{T}}$, the two models can be distinguished by considering

$$
\begin{aligned}
\beta_{A}^{T}= & {\left[b w_{R_{1}} b w_{R_{2}} b w_{R_{3}} b w_{C_{1}} b w_{C_{2}} b w_{C_{3}} a w_{R_{1}} a w_{R_{2}}\right.} \\
& \left.a w_{R_{3}} a w_{C_{1}} a w_{C_{2}} a w_{C_{3}}\right]
\end{aligned}
$$

under the hypothesis that the weights differ from rows to columns and

$$
\beta_{0}^{\mathrm{T}}=\left[\mathrm{bw}_{1} \mathrm{bw}_{2} \mathrm{bw}_{3} \mathrm{aw}_{1} \mathrm{aw}_{2} \mathrm{aw}_{3}\right]
$$

under the hypothesis that row weights and column weights are equal. To answer the second question, if the first $\mathrm{H}_{0}$ is accepted, the vector $\beta_{1}^{\mathrm{T}}=[2 \mathrm{ba}]$ would be used for the test of one weight vs. three.

Throughout the development of the paper, the simplifying assumption has been made that replica- tions could be obtained either between or within subjects. Thus, each subject was assumed to have not only the same psychophysical law in its form, but also the same values of the parameters. If, however, such individual difference effects are to be seriously considered, provision must be made in the various analyses. Space limitation precludes detailed treatment, but the general approach can be exemplified. For the regression model, taking as our example the case of the additive psychological law with linear psychophysical laws, let $\mathbf{R}_{\mathrm{i}}=\mathbf{X}_{\mathrm{i}}^{\mathrm{T}} \boldsymbol{\beta}_{\mathrm{i}}+\mathbf{e}$ be the model for subject $i$. Then the overall regression model can be written with the overall response vector containing all the individual subject response vectors, the $\mathbf{R}_{\mathrm{i}}$, strung together as one column vector, the overall parameter vector containing parameters $2 b_{i}, a_{1 i}$, and $a_{2 i}$ for each subject for the larger model and parameters $2 b_{i}$ and $a_{i}$ for each subject for the smaller model, and the overall $\mathbf{X}^{\mathbf{T}}$ matrix laid out appropriately. This model can be tested against the model with only group parameters as a test for the presence of individual differences.

When the procedures involve testing the hypothesis that the row means equal the column means, and the replications are between subjects, the analysis of variance in Table 6 should be modified. Suppose, for example, the model for subject $k$ is

$$
R_{i j k}=c_{1 k} S_{i}^{n_{1 k}}+c_{2 k} T_{j}^{n_{2 k}}+b_{k}
$$

and we wish to test the null hypothesis that $c_{1 k}=$ $c_{2 k}, n_{1 k}=n_{2 k}$, for all $k$. This null hypothesis implies that the row means will equal the column means. The numerator mean square for the appropriate $F$ test will be as given in Table .6. According to the model, there should be no Row by Column by Subjects interaction, although a Row by Subjects and a Column by Subjects interaction are expected. So the $R \times C \times S$ mean square furnishes an error term for the test of the null hypothesis. The presence of subject effects can be tested by testing the effects of subjects and the $\mathrm{R} \times \mathrm{S}$ and the $\mathrm{C} \times \mathrm{S}$ interactions, again using the $\mathrm{R} \times \mathrm{C} \times \mathrm{S}$ interaction as the error term. With respect to these tests of subject effects, assuming that the basic model is correct, there can be no $\mathrm{R} \times \mathrm{C} \times \mathrm{S}$ interaction, so the usual negative bias involved in testing $\mathrm{R} \times \mathrm{S}$ or $\mathrm{C} \times \mathrm{S}$ against $\mathrm{R} \times \mathrm{C} \times \mathrm{S}$ is avoided. If concern for the basic assumption of the model exists, a further check on the model could be conducted by testing for nonadditivity. Following Tukey (1949), the RCS interaction sum of squares could be partitioned into a Linear by Linear by Linear component on $1 \mathrm{df}$ which would be tested against the residual mean square.

The present use of multiple regression procedures in hypothesis testing and parameter estimation in the functional measurement-information integration 
theory approach to psychophysics has great versatility. It is adaptable to many of the cognitive algebras of interest and is compatible with standard computer library functions for regression analysis. It should provide the statistical basis for a considerably more detailed elaboration of psychophysical laws within the information integration theory framework.

\section{REFERENCE NOTE}

1. Bogartz, R. S. Testing the equality of means or the proportionality of sets of effects from factor to factor in a fixed-effects experimental design. Unpublished manuscript, 1979.

\section{REFERENCES}

Anderson, N. H. Algebraic models in perception. In E. C. Carterette \& M. P. Friedman (Eds.), Handbook of perception (Vol. 2). New York: Academic Press, 1974
Bogartz, R. S., \& Wackwitz, J. H. Polynomial response scaling and functional measurement. Journal of Mathematical Psychology, 1971, 8, 418-443.

Curtis, D. W., \& Rule, S. J. Judgment of duration relations: Simultaneous and sequential presentation. Perception \& Psychophysics, 1977, 22, 578-584.

Hartey, H. O. The modified Gauss-Newton method for the fitting of nonlinear regression functions by least squares. Technometrics, 1961, 3, 269-280.

Mood, A. M., \& GRAYBILl, F. A. Introduction to the theory of statistics (2nd ed.). New York: McGraw-Hill, 1963.

Tukey, J. W. One degree of freedom for nonadditivity. Biometrics, 1949, 5, 232-242.
(Received for publication August 8, 1979;
revision accepted December 28, 1979.) 